\title{
Tail Pinch Induced Stress-Arousal Facilitates Brain Stimulation Reward
}

\author{
R. J. KATZ' AND KEVIN ROTH \\ Mental Health Research Institute, Department of Psychiatry \\ University of Michigan Medical Center, Ann Arbor, MI 48109
}

(Received 27 July 1978)

\begin{abstract}
KATZ, R. J. AND K. ROTH. Tail pinch induced stress-arousal facilitates brain stimulation rew'ard. PHYSIOL. BEHAV. 22(1) 193-194, 1979.-Adult male Sprague Dawley rats with chronic access to self stimulation were subjected to handling, tail pinch, or left undisturbed. Tail pinch increased responding for positive reinforcement while the other conditions did not. The stress related properties of tail pinch may therefore facilitate responding in the present and other situations.
\end{abstract}

Arousal Brain stimulation ICS Self stimulation Stress Tail pinch

TAIL PINCH is a psychophysically complex stimulus known to produce stress and arousal. Behavioral concomitants of stimulation include oral stereotypy, feeding, maternal behavior, learning, and aggression $[1,2,6,7,9]$. Neither the precise ecological determinants of this motivated state, nor the manner by which tail pinch affects behavior are well established. One possible explanation for tail pinch effects suggests arousal or possibly stress related consequences of tail pinch cause a general increase in prepotent behaviors [11]. If this hypothesis is correct then the boundaries of motivational plasticity associated with tail pinch remain to be determined. Would tail pinch, for example, affect a positively reinforced task using brain stimulation? As one of a series of studies on stress and reinforcement mechanisms, we have subjected chronically self stimulating rats to tail pinch. Our findings suggest that brain stimulated reward is in fact responsive to tail pinch stimulation. Moreover, comparisons across conditions indicate the facilitating effects of tail pinch may be due to stress.

\section{METHOD}

\section{Animals}

Nine adult male Sprague Dawley rats $(350-500 \mathrm{~g}$ each) were obtained locally (Charles River Farms, Portage, MI) and maintained on ad lib food (Teklad $4.0 \%$ fat diet S-0836) and tap water, and automatically programmed lighting cycles of $12 \mathrm{hr}$ (lights on $=8: 00-20: 00 \mathrm{hr}$ ).

\section{Apparatus and Procedure}

The self stimulation design of Wolf et al. [12] was employed for all testing. Animals were implanted under sodium pentobarbital (Nembutal $50 \mathrm{mg} / \mathrm{kg}$ ) anesthesia with unipolar $0.025 \mathrm{~cm}$ nichrome wire electrodes insulated to the tip. Each electrode was attached to a head mounted brass brushing, and secured to the skull with stainless steel screws and acrylic dental dement. Each animal's cage (individual $25 \times 18 \times 17$ $\mathrm{cm}$, of stainless steel) contained a $14 \times 16 \mathrm{~cm}$ steel plate located $14 \mathrm{~cm}$ from the floor which served as a contact plate and manipulandum. Upwards directed pressture of approximately $20 \mathrm{~g}$ applied to the plate resulted in the completion of a circuit for which the cage floor served as ground. A pulse of $0.3 \mathrm{sec}$ sinusoidal $60 \mathrm{cps}$ current was delivered through the brushing to the rat for each response. Current ranged from $50-300 \mu \mathrm{A}$ in intensity for individual rats, and was delivered through a series of capacitors and high resistances and across an isolation transformer to maintain approximately constant current conditions. All electrodes were aimed at the medial forebrain bundle (coordinates from Bregma $=-5.0$, 1.0-8.0) and were verified histologically in a subsample of 4 rats. A minimum of 2 weeks access to the apparatus and behavioral contingency preceded testing. During this period, animals remained undisturbed except for daily weighing and periodic cage maintenance (i.e. replacement of food, water, bedding). The animals were examined for self stimulation during a period of normal quiescence (for all animals 11:00 14:00 hr), and 3 measurements separated by at least $24 \mathrm{hr}$ were taken for each animal. Rates of self stimulation behavior were taken for $1 \mathrm{hr}$ during normal undisturbed quiescence, immediately after weighing, and after tail pinch. For the latter, a rubber dam forceps closed to the first notch was manually applied to the tail $3-4 \mathrm{~cm}$ from its base for $30 \mathrm{sec}$. This produced a mild nontraumatic pressure when tested on human fingers. Order of treatments was random with respect to animals.

'Send reprint requests to $\mathbf{R}$. J. Katz. 


\section{RESULTS}

Table 1 indicates that the testing period is normally a time characterized by low rates of self-stimulation. Visual observations of the rats indicated that they were not sleeping. It may also be seen in Table 1 that weighing and tail pinch both increased self stimulation. Repeated measures analysis of variance [4] revealed a significant effect across groups $(\mathrm{F}(2,16)=4.17, p<0.05)$. Post hoc comparisons by Duncan's multiple range test $[3]$ indicated significant $(\rho<0.05)$ differences for quiescence and tail pinch, and handling and tail pinch (observed values were $23.8,20.8$ ). No significant difference was observed in comparing quiescence and weighing (observed value $=3.0, p>0.05$ ). Therefore, tail pinch significantly elevated behavior from either of two baseline conditions which did not themselves differ. (We have had occasion to test 2 adrenalectomized animals using the above procedure, and our results were essentially similar in all respects. Rates after behavior quiescence, handling and tail pinch were 0,0 , and $600 \pm 420$. These preliminary results suggest an extra-adrenal mediation of behavioral change.)

TABLE 1

HOURLY RATES OF SELF STIMULATION UNDER THREE CONDITIONS (MEAN \pm SEM)

\begin{tabular}{cc}
\hline Quiescence & $187 \pm 187$ \\
Handling & $276 \pm 153$ \\
Tail Pinch & $900 \pm 482$ \\
\hline
\end{tabular}

Mean and standard error calculated across animals. All analysis is based upon within animals changes.

Linear regression analysis of tail pinch induced self stimulation upon previous $24 \mathrm{hr}$ self stimulation scores indicated a correlation coefficient of $r=0.92(p<0.05$ by Fisher's transformation $t=6.3$ ). High baseline responders therefore were the most active responders to tail pinch. This may be interpreted as an overall individual responsiveness which is evident across situations but specific to animals, and also as a further indicator of the specificity of the manipulation with respect to reward.

\section{DISCUSSION}

Tail pinch has been reported to affect a variety of behaviors. Our results confirm and extend the motivational generality of the phenomenon. These findings are consistent with other reports suggesting stressors may affect brain stimulated reward $[5,8]$. For example, a buzzer paired with shock selectively reinstated a bar-pressing habit in rats which were trained to respond for stimulation to tegmental sites, and subsequently extinguished [5]. Moreover, normally quiescent monkeys sought hypothalamic stimulation strictly in conjunction with emotionally arousing stimulation (i.e. the presentation of a toy snake or dominant animal) [8]. Other findings, e.g. the reversal of conditioned emotional suppression by brain stimulation reward [3] also indirectly suggest that selected CNS areas are rewarding in response to stress. They may represent a "relief' producing system which requires prior emotional activation. In the present report animals again sought stimulation after stress.

Our results suggest that merely arousing an animal i.e. through weighing produces a minor facilitation of responding which actually is not significantly different from a control baseline. On the other hand, however, the consequences of tail pinch upon reward are substantially higher than those associated with resting or handling. It might be argued that handling is perhaps less arousing, or, more likely arousing but less stressful. The stress related consequences of tail pinch may therefore be important for the observed facilitation. These findings extend the generality of tail pinch related behavioral change, stress, and behavior.

\section{ACKNOWLEDGMENT}

Dr. Katz was supported by postdoctoral grant MH07417 from the National Institute of Mental Health through the Mental Health Research Institute. Giulio Baldrighi performed all surgery; Esther Washington contributed editorial assistance.

\section{REFERENCES}

1. Antelman, S. M. and H. Szechtman. Tailpinch induces eating in sated rats which appears to depend on nigro striatal dopamine. Sicience 189: 731-733, 1975.

2. Antelman, S. M., H. Szechtman, P. Chin and A. E. Fisher. Tailpinch induced eating, gnawing, and licking behavior: Dependence on the nigro striatal dopamine system. Brain Res. 99: $319-337,1975$.

3. Brady, J. V. and D. C. Conrad. Some effects of limbic system self stimulation upon conditioned emotional behavior. $J$. comp physiol. Prychol. 53: 123-137, 1960.

4. Bruning, J. L. and B. L. Kintz. Computational Hamalbosh of Statistics, 2nd ed. Glenview: Scott Foresman, 1977.

5. Deutsch, J. A. and C. I. Howarth. Evocation by fear of a habit learned for electrical stimulation of the brain. Science 136: 1057-1058, 1962

6. Katz, R. Tail pinch arousal or aversion. Science 201: 840-841, 1978 .
7. Koob, G. F., P. J. Fray and S. D. Iversen, Tail pinch stimulation: sufficient motivation for learning. Science 194: 637-639. 1976.

8. Maxim, P. Self-stimulation of a hypothalamic site in response to tension or fear. Physiol. Behav. 18: 197-201, 1977.

9. Szechtman, D., H. I. Siegel, J. S. Rosenblatt and B. R Komisaruk. Tail pinch facilitates onset of maternal behavior in rats. Physiol. Behav. 19: 807-809, 1977.

10. Wallach, M. B., M. Dawber, M. McMahon and C. Rogers. A new anorexigen assay: stress-induced hyperphagia in rats. Pharmac. Biochem. Behav. 6: 529-531, 1977.

11. Valenstein, E. S. Behavior elicited by hypothalamic stimulation, a prepotency hypothesis. Brain Beha'. Elolui. 2(4): 295 $316,1969$.

12. Wolf, G., L. V. DiCara and W. Simpson. The contact method: a simple technique for electrical self stimulation without external leads. Phrsiol. Behar. 11: 721-723, 1973 